\title{
Inference on population history and model checking using DNA sequence and microsatellite data with the software DIYABC (v1.0)
}

\author{
Jean-Marie Cornuet ${ }^{1}$, Virgine Ravigné ${ }^{2}$, Arnaud Estoup ${ }^{1 *}$
}

\begin{abstract}
Background: Approximate Bayesian computation $(A B C)$ is a recent flexible class of Monte-Carlo algorithms increasingly used to make model-based inference on complex evolutionary scenarios that have acted on natural populations. The software DIYABC offers a user-friendly interface allowing non-expert users to consider population histories involving any combination of population divergences, admixtures and population size changes. We here describe and illustrate new developments of this software that mainly include (i) inference from DNA sequence data in addition or separately to microsatellite data, (ii) the possibility to analyze five categories of loci considering balanced or non balanced sex ratios: autosomal diploid, autosomal haploid, X-linked, Y-linked and mitochondrial, and (iii) the possibility to perform model checking computation to assess the "goodness-of-fit" of a model, a feature of $A B C$ analysis that has been so far neglected.

Results: We used controlled simulated data sets generated under evolutionary scenarios involving various divergence and admixture events to evaluate the effect of mixing autosomal microsatellite, mtDNA and/or nuclear autosomal DNA sequence data on inferences. This evaluation included the comparison of competing scenarios and the quantification of their relative support, and the estimation of parameter posterior distributions under a given scenario. We also considered a set of scenarios often compared when making $A B C$ inferences on the routes of introduction of invasive species to illustrate the interest of the new model checking option of DIYABC to assess model misfit.

Conclusions: Our new developments of the integrated software DIYABC should be particularly useful to make inference on complex evolutionary scenarios involving both recent and ancient historical events and using various types of molecular markers in diploid or haploid organisms. They offer a handy way for non-expert users to achieve model checking computation within an $A B C$ framework, hence filling up a gap of $A B C$ analysis. The software DIYABC V1.0 is freely available at http://www1.montpellier.inra.fr/CBGP/diyabc.
\end{abstract}

\section{Background}

Natural populations are often characterized by complex demographic histories. Their effective sizes and ranges change over time leading to fission and fusion processes that leave signatures on their genetic constitution and structure. One promising prospect of current biology is that molecular data will help us to reveal the complex demographic processes that have acted on populations. The extensive availability of different molecular markers

\footnotetext{
* Correspondence: estoup@supagro.inra.fr

'INRA, UMR CBGP (INRA/IRD/Cirad/Montpellier SupAgro), Campus international de Baillarguet, CS 30016, F-34988 Montferrier-sur-Lez cedex,
} France

(c) 2010 Cornuet et al; licensee BioMed Central Ltd. This is an Open Access article distributed under the terms of the Creative Commons Attribution License (http://creativecommons.org/licenses/by/2.0), which permits unrestricted use, distribution, and reproduction in any medium, provided the original work is properly cited. and increased computer power has promoted the development of inferential methods and associated software that have begun to fulfil these expectations [1,2].

Approximate Bayesian computation $(\mathrm{ABC})$ is a recent flexible class of Monte-Carlo algorithms for performing model-based inference [3]. Estimations associated with demographic and genetic models often imply a full likelihood calculation, which is difficult for complex evolutionary scenarios. ABC methods bypass exact likelihood calculations by using summary statistics and massive computer simulations and make it possible to handle large data sets, such as data for hundreds of individuals genotyped at tens of microsatellite loci. The 
development of $\mathrm{ABC}$ has hence generated a sharp increase in the complexity of models used in various fields $[4,5]$. ABC methods were recently successfully used to make inference on complex models in population and evolutionary biology [6-13], infectious disease epidemiology [14] and system biology [15]. Such inferences mainly include model selection among a finite set of models (evolutionary scenarios) and inferences on the posterior distribution of the parameter of interest under a given model. Whereas several studies have now shown that parameter posterior distributions inferred by $A B C$ are similar to those provided by full-likelihood Bayesian approaches [16-19], the approach is still in its infancy and continues to evolve, and to be improved (reviewed in $[4,5,20,21])$. In statistical analysis assessing the "goodness-of-fit" of a model (here an evolutionary scenario) with respect to a "real" data set is termed model checking. If a (selected) model has a good fit then the observed data set should look plausible under the posterior predictive distribution of the model [22]. Although useful when doing inferences, model checking is a feature of $A B C$ analyses that has been so far neglected ([5]; but see [23-25]).

Until recently, the $A B C$ approach has remained inaccessible to most biologists because of the complex computations involved. Since 2008, several ABC softwares have been proposed to provide solutions to non-specialist users [26-32]. Cornuet et al. [26] developed the software DIYABC in which a user-friendly interface helps non-expert users to perform historical inference using $A B C$. DIYABC allows considering complex population histories involving any combination of population divergences, admixtures and population size changes, with population samples potentially collected at different times. DIYABC can be used to compare competing evolutionary scenarios and quantify their relative support, and estimate parameters for one or more scenarios. Eventually, it provides a way to evaluate the amount of confidence that can be put into the various estimations. So far, DIYABC applied only to independent autosomal microsatellite data and did not offer users to achieve model checking computation.

This article describes new developments of DIYABC that mainly include (i) the extension of $\mathrm{ABC}$ analysis to DNA sequence data in addition or separately to microsatellite data and (ii) the possibility to proceed model checking computation to assess the "goodness-of-fit" of a model within an ABC framework. We used controlled simulated data sets generated under complex evolutionary scenarios to evaluate the interest of mixing autosomal microsatellite, mtDNA and/or nuclear autosomal DNA sequence data. We also used a set of scenarios often considered when making $\mathrm{ABC}$ inferences on the routes of introduction of invasive species to illustrate the interest of the model checking option of DIYABC to assess model misfit.

\section{Methods}

New implementations in DIYABC V1.0

The new version of the software allows the treatment of haploid in addition to diploid data. Five categories of loci (either microsatellites or DNA sequences) can now be analyzed together or separately: autosomal diploid, autosomal haploid, X-linked, Y-linked and mitochondrial. X-linked loci can be used for a haplo-diploid species in which both sexes have been sampled. The data for each type of markers may have been obtained from the same or different individuals. Balanced or non balanced sex ratios can be considered.

Four different mutation models can be chosen for DNA sequence data. For all mutation models, insertiondeletion mutations are not considered mainly because there does not seem to be much consensus on this topic. Concerning substitutions, we have implemented the following models: the Jukes-Cantor [33] one parameter model, the Kimura [34] two parameter model, the Hasegawa-Kishino-Yano [35] and the Tamura-Nei [36] models. The last two models include the ratios of each nucleotide as parameters. However, in order to reduce the number of parameters, these ratios have been fixed to values observed in the data for each DNA sequence locus. Consequently, this leaves two and three variable parameters for the Hasegawa-Kishino-Yano (HKY) and Tamura-Nei (TN), respectively. Summary statistics can be chosen for DNA sequence data among a set of 14 statistics detailed in the notice document available at http://www1.montpellier.inra.fr/CBGP/diyabc. As for microsatellite loci, DNA sequence summary statistics are averaged for a type of sequence loci (e.g. nuclear DNA sequence loci). This allows reducing the total number of summary statistics as the latter may quickly increase when considering summary statistics independently for each sequence locus.

With respect to microsatellite loci, the possibility of uneven insertion/deletion events (i.e. allele lengths are sometimes not multiple of the motif length implying that there has been single nucleotide insertion-deletion mutations in the flanking regions of microsatellites [37]) is now better taken into account in inferences as this type of mutation events is not considered anymore as a nuisance parameter but can be estimated by considering a mean mutation rate (mean $\mu \mathrm{SNI}$ ) drawn from various prior distributions and some individual locus mutation rates drawn from some Gamma distribution with mean $=$ mean $\mu \mathrm{SNI}$.

A new option called "evaluate scenario-prior combination" allows checking whether some of the models together with the chosen prior distributions have the 
potential to generate a subset of summary statistics close to the observed summary statistics (i.e. the target statistics obtained from the data set on which one wants to make inferences). In the first analysis proposed by this option, a principal component analysis is performed in the space of summary statistics on at most 10,000 simulated data sets and the target (observed) data set is added on each plane of the analysis in order to evaluate how the latter is surrounded by the simulated data sets. In addition to this global approach, there is a second one in which each summary statistic of the observed data set is ranked against those of the simulated data set. This second analysis helps finding which aspects of the model (including prior) is problematic. For instance, a grossly underestimated genetic distance (in simulated data sets compared to the observed one) may suggest a misspecification of the prior distribution of a divergence time between two populations or of the mean mutation rate of the markers. To our experience, using this new option before running a full $A B C$ treatment with DIYABC is a convenient and easy way to reveal noticeable misspecification of prior distributions and/or models (see Additional file 1 for an illustration).

Following Gelman et al. ([22] pp 159-163), we implemented a new option in DIYABC V1.0, called "model checking", to measure the discrepancy between a combination of a model and parameter posterior distributions and a "real" data set by considering various sets of test quantities. These test quantities can be chosen among the large set of $A B C$ summary statistics proposed in DIYABC V1.0. Details regarding these new computations are given below in the methods and results sections entitled Model checking.

DIYABC V1.0 was written in Delphi 2009 and runs under a 32-bit Windows operating system. It is worth stressing that this new version of the software was recoded in order to use a multithread technology allowing the exploitation of multicore/multiprocessor computers. This is especially useful when building the reference table and for several other intensive computation steps, such as the multinomial logistic regression. Such improvements allow a substantial gain of speed for $\mathrm{ABC}$ treatments when using multicore/multiprocessor computers, which now are found in most biology research laboratories.

\section{Mixing microsatellite, mtDNA and/or nuclear DNA sequence data}

In order to evaluate the interest of mixing microsatellite loci with mtDNA and/or nuclear DNA sequence data, we used simulated data sets generated under three complex evolutionary scenarios similar to those presented in Cornuet et al. [26]. These scenarios involved different number of divergence and admixture events that occurred at recent to ancient times (see Figure 1). We evaluated the potential of different types of data sets (ten autosomal microsatellite loci, one mtDNA sequence of 1,000 nucleotides, five nuclear autosomal DNA sequences of 1,000 nucleotides each, and all combinations of two and three types of markers) to compare the three competing scenarios and estimate parameters under each scenario.

Prior distributions of demographic parameters were as followed: Uniform[10; 10000] for effective population sizes (similar for all populations), Uniform $[1 ; 100]$ for $t 1$, Uniform [100; 1000] for $t 2$, Uniform [5000; 50000] for $t 3, t 3 a$, and $t 4$ (with $t 4>t 3$ ), Uniform [50000; 500000] for $t 5$, and Uniform $[0.1 ; 0.9]$ for $r 1$ and $r 2$. For microsatellite markers, the ten loci were assumed to follow a generalized stepwise mutation model (GSM [37]) with two parameters: the mean mutation rate (mean $\mu$ ) and the mean parameter of the geometric distribution of the length in number of repeats of mutation events (mean $P)$ drawn from Uniform[10-4; 10-3] and Uniform[0.1; $0.3]$ prior distributions, respectively. Each locus has a possible range of 40 contiguous allelic states and was characterized by individual $\mu_{l o c}$ and $P_{l o c}$ values drawn from $G a m m a($ mean $=$ mean $\mu$ and shape $=2)$ and Gamma(mean $=$ mean $P$ and shape $=2$ ) distributions, respectively [12]. For DNA sequence loci (one mtDNA locus and five nuclear DNA loci), the sequences were assumed to follow the two parameter model of Kimura [34] with a fraction of constant sites (those that cannot mutate) fixed to $10 \%$ and the shape parameter of the Gamma distribution of mutations among sites equal to 2. For each sequence locus $(1,000$ nucleotide per sequence), the mean mutation rate per nucleotide and generation was drawn in a Uniform $\left[10^{-8} ; 10^{-7}\right]$ and a Uniform $\left[10^{-9} ; 10^{-8}\right]$ for the mtDNA and nuclear sequences, respectively [53].

The summary statistics for microsatellite loci were the mean number of alleles, expected heterozygosity [38] and allele size variance per population, $F_{\mathrm{ST}}$ values and genetic distance $(\delta \mu)^{2}$ between pairs of populations $[39,40]$ and the maximum likelihood estimate of admixture proportion [41]. The summary statistics for DNA sequence loci were (i) the number of distinct haplotypes, the number of segregating sites, the mean pairwise difference, the variance of the number of pairwise differences (all statistics computed within each sample), (ii) the number of distinct haplotypes, the number of segregating sites (all statistics computed in samples pooled by pair), and (iii) the $F_{\mathrm{ST}}$ between pairwise samples (computed as in [42]) and an adaptation for sequence data of the maximum likelihood estimate of admixture proportion of Choisy et al. [41]. Mean values of such statistics were computed over loci grouped by category (microsatellites, nuclear DNA sequences and mitochondrial DNA sequence). 
For each combination of marker type, we simulated $10^{6}$ data sets for each of the three competing scenarios. For each competing scenario, we simulated 500 test data sets (i.e. pseudo-observed data sets) drawing demographic and marker parameter values in the same distributions as those used to generate the reference table (see legend of Figure 1). For model comparison, we estimated the posterior probabilities of the competing scenarios using a polychotomous logistic regression on the $1 \%$ of simulated data sets closest to the observed data set [26]. Posterior probabilities of the three scenarios were used to compute type I and II errors in the choice of each scenario. For instance, let us consider the estimation of type I and type II errors when choosing scenario 2 as the true scenario. To do so, we simulate 500 data sets according to scenario 1,2 and 3 . Then we count the proportion of times that scenario 2 has not the highest posterior probability among the three competing scenarios when it is the true scenario (type I error, estimated from test data sets simulated under scenario 2) or the proportion of times that scenario 2 has highest posterior probability when it not the true scenario (type II error, estimated from test data sets simulated under scenarios 1 and 3).

We then estimated the posterior distributions of parameters under the most complex scenario (i.e. scenario 1 ) using a local linear regression on the $1 \%$ closest simulated data sets and applying a logit transformation to parameter values $[3,26]$. We evaluated the precision of parameter estimation by computing the median of the absolute error divided by the true parameter value of the 500 pseudo-observed data sets simulated under scenario 1 using the median of the posterior distribution as point estimate (RMAE). All computations were processed using DIYABC V1.0.

\section{Model checking}

A combination of a model and parameter posterior distributions is acceptable only if the observed data look similar to replicated data generated under this modelposterior combination (i.e. under the posterior predictive distribution; $[5,20])$. To put it another way, the observed data should look plausible under the posterior predictive distribution. This is really a self-consistency check: an observed discrepancy can be due to model misfit (demographic and/or marker models) or chance. Following Gelman et al. ([22] pp 159-163), we implemented an option in DIYABC V1.0 to evaluate the discrepancy between a model-posterior combination and a target (observed) data set by considering various sets of test quantities. These test quantities are chosen among the set of ABC summary statistics proposed in DIYABC V1.0 (see the notice document available at http://www1. montpellier.inra.fr/CBGP/diyabc for an illustration). For

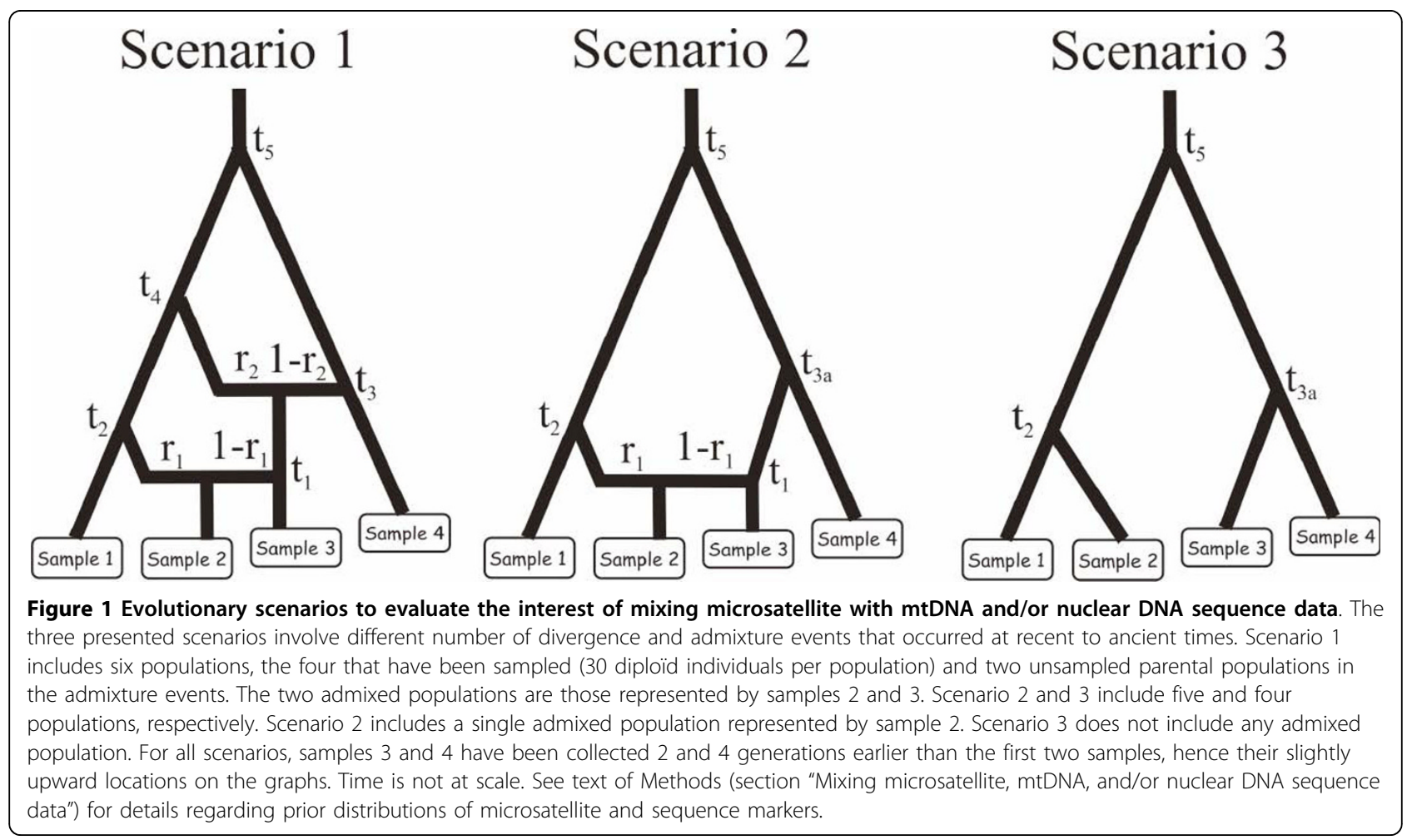


each test quantities ( $t$ corresponding to the chosen summary statistics), a lack of fit of the observed data with respect to the posterior predictive distribution can be measured by the cumulative distribution function values of each test quantities defined as $\operatorname{Prob}\left(t_{\text {simulated }}<t_{\mathrm{ob}}\right.$ served). Tail-area probability, or $p$-value, can be easily computed for each test quantities as $\operatorname{Prob}\left(t_{\text {simulated }}<t_{\mathrm{ob}}\right.$ served $)$ and 1.0 - Prob $\left(t_{\text {simulated }}<t_{\text {observed }}\right)$ for Prob $\left(t_{\text {simu- }}\right.$ lated $\left.<t_{\text {observed }}\right) \leq 0.5$ and $>0.5$, respectively [22]. Such $p$-values represent the probability that the replicated data (simulated ABC summary statistics) could be more extreme than the observed data (observed ABC summary statistics). Too many observed summary statistics on the tails of distributions would cast serious doubts on the adequacy of the model-posterior combination. Because $p$-values are computed for a number of test statistics, we used the method of Benjamini and Hochberg [43] to control the false discovery rate (see [44] for a comparative study of several methods dealing with false discovery rate control and [23] for an application in the context of an $\mathrm{ABC}$ study). An alternative way to combine $p$-values across test statistics has been recently proposed [25].

One complication with inferences using $\mathrm{ABC}$ is that at least some and sometimes all summary statistics used as tests quantities have already been used during the inference steps (model discrimination and estimation of parameters). There is a risk of over-estimating the quality of the fit by using the same statistics twice. This problem which clearly arises within an $\mathrm{ABC}$ framework is actually a general one in statistical inference. As underlined in many text books in statistics (e.g. $[22,45]$ and see [5]), it is advised against performing model checking using information that have already been used for training (i.e. model fitting). Optimally, model checking should be based on test quantities that do not correspond to the summary statistics that have been used for previous inferential steps; this is naturally possible with DIYABC as the package propose a large choice of summary statistics. The choice of the two sets of statistics remains a difficult issue that still needs to be thoroughly investigated (and that we will not investigate here). In practice, one could advise users to choose the set of statistics for the model discrimination and parameter estimation step and the set of statistics for the model checking step before they embark on the first step. Moreover, it seems sensible that both sets include statistics describing genetic variation both within and between populations.

To illustrate this new model checking option of DIYABC V1.0, we have chosen a set of basic scenarios considered when making $\mathrm{ABC}$ inferences on the routes of introduction of invasive species $[46,47]$. We considered three models in which two invasive populations originate from the same source population. These populations may be related through three different scenarios: the independent introduction scenario, the serial introduction scenario and the unsampled population scenario (Figure 2). In the independent or serial introduction scenarios, all the populations concerned were sampled, but in the unsampled population scenario, the two invasive populations were founded independently from an undetected and hence unsampled population, itself introduced from the source. It is worth stressing that although previous studies have shown that some invasive populations may remain undetected but may play important role in the invasion dynamics of some species $[48,49]$, the unsampled population scenario is often not considered. If only the traditional independent and serial introduction scenarios are compared, a "real" data set obtained under the unsampled population scenario will erroneously fit one of the two competing scenario with often a high posterior probability (see results section and [47]). Here we used a single, randomly chosen, pseudo-observed test data set simulated under the unsampled population scenario to illustrate the interest of the model checking option of DIYABC.

Standard ABC analyses (estimation of model probabilities and of parameter posterior distributions) were first performed on the above test data set as described previously (i.e. in the section "Mixing microsatellite, mtDNA and/or nuclear DNA sequence data"). We drew parameter values from the prior distributions described in the legends of Figure 2 and used the summary statistics described in Table 1. Model checking computations were then processed by simulating 10,000 data sets under each studied model-posterior combination, with sets of parameter values drawn with replacement among the 10,000 sets of the posterior sample. We computed two groups of test quantities: a first group of summary statistics already used for model discrimination and estimation of parameter posteriors and a second group of summary statistics not previously used for inferences. Each observed summary statistics was then ranked and given cumulative distribution function values among the corresponding sample of summary statistics obtained through the above simulation, providing an estimation of $p$-value for each summary statistics. In addition, a principal component analysis (PCA) was performed in the space of summary statistics. Principal components were computed considering 10,000 data sets simulated with parameter values draw from the prior. Then the target (observed) data set as well as the 1,000 data sets simulated from the posterior distributions of parameters were added to each plane of the PCA. If the model-posterior combination fits well the observed data set, one should see on each PCA plane a wide cloud of data sets simulated from the prior, with the observed data set in 


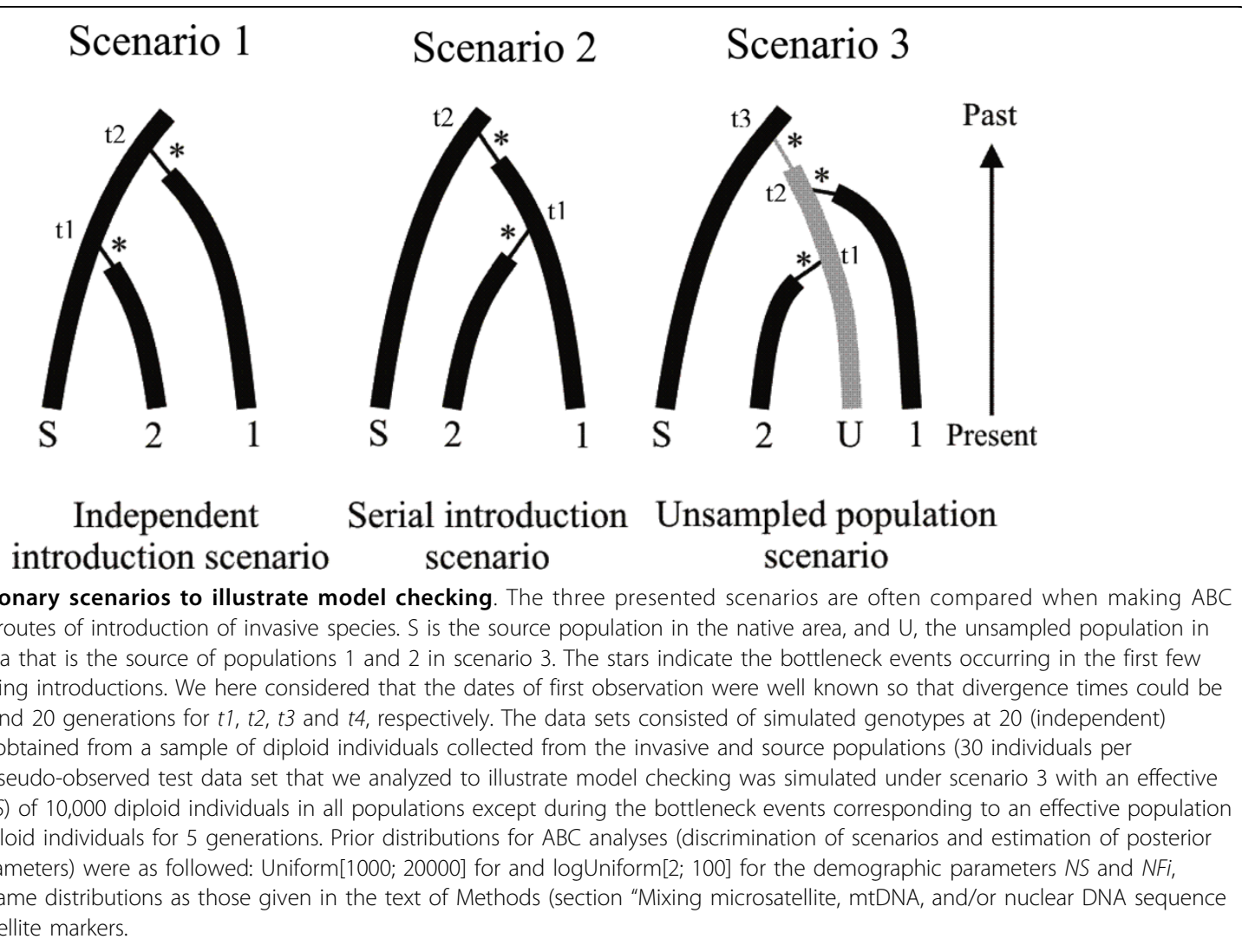

the middle of a small cluster of data sets generated from the posterior predictive distribution. All computations and illustrations were processed using DIYABC V1.0.

\section{Results and Discussion \\ Mixing microsatellite, mtDNA and/or nuclear DNA sequence data}

Results dealing with the discrimination among a finite set of competing complex scenarios are summarized in Figure 3. When considering the confidence in scenario choice for each type of markers taken separately, we found that the lowest error rates were obtained for different types of markers depending on the type of error and scenario considered. The lowest type I error rates were obtained with the nuclear sequences for scenarios 1 and 2, and microsatellites or mtDNA for scenario 3 . The lowest type II error rates were obtained with mtDNA for scenario 1, the nuclear sequences for scenario 2 and microsatellites for scenario 3 . Some differences in error rates between markers were small, however, and hence not significant using Fisher exact tests (e.g. type II errors for scenario 1 were equal to 0.023 and 0.024 for mtDNA and nuclear sequences, respectively). MtDNA displayed contrasted error rates depending on the scenario considered, with sometimes large error values; for instance a type I error of 0.458 for scenario 1 and a type II of error 0.225 for scenario 2 . These large error rates were probably due to the fact that mtDNA data correspond to a single locus and hence to a single gene genealogy subject to substantial stochastic variation [50]. Adding sequence data (mtDNA or nuclear DNA) to microsatellite data globally decreased type I errors (especially for scenario 2 for which type I error was two times lower) and type II errors (especially for scenario 1 for which type I error was two times lower). For all three scenarios, the lowest type I and II error values were obtained when combining the three types of markers.

Results dealing with the estimation of parameters under scenario 1 are summarized in Figure 4. Whatever the type and combination of markers, the molecular data provided substantial information for all parameters except the divergence times $t 1, t 2$ and $t 4$ for which the level of information remained low. For the latter parameters the relative median absolute errors (RMAE) were only slightly lower than those computed as base level using only the prior information on parameters (blue bars in Figure 4). This is not surprising since t1 corresponds to a very recent time of admixture $(<100$ generations) and $\mathrm{t} 2$ or $\mathrm{t} 3$ correspond to divergence times for which one of the two diverging populations has not been sampled. 
Table 1 Model checking for introduction scenarios 1, 2 and 3

\begin{tabular}{|c|c|c|c|c|c|}
\hline & \multirow[b]{2}{*}{ Test quantity $(t)$} & \multirow[b]{2}{*}{ Observed value } & \multicolumn{3}{|c|}{ Probability $\left(t_{\text {simulated }}<t_{\text {observed }}\right)$} \\
\hline & & & Scenario 1 & Scenario 2 & Scenario 3 \\
\hline Test quantities & NAL_S & 13.6000 & 0.7275 & 0.2871 & 0.6235 \\
\hline corresponding & NAL_1 & 3.4000 & 0.7542 & $0.9865\left(^{*}\right)$ & 0.4252 \\
\hline to thesummary & NAL_2 & 3.6500 & 0.6455 & 0.4102 & 0.4761 \\
\hline statistics used & HET_S & 0.8429 & 0.5621 & 0.2471 & 0.4488 \\
\hline to discriminate & HET_1 & 0.5151 & 0.4938 & $0.9890(*)$ & 0.4339 \\
\hline among & HET_2 & 0.5725 & 0.9125 & 0.9188 & 0.8221 \\
\hline scenarios and & MGW_S & 0.8242 & 0.3593 & 0.7656 & 0.5230 \\
\hline compute & MGW_1 & 0.4072 & 0.3782 & 0.6713 & 0.4524 \\
\hline parameter & MGW_2 & 0.4834 & 0.6117 & 0.8499 & 0.7297 \\
\hline posterior & FST_S_1 & 0.2170 & 0.7882 & $0.0371\left(^{*}\right)$ & 0.8105 \\
\hline \multirow[t]{2}{*}{ distributions } & FST_S_2 & 0.2050 & 0.6180 & 0.4606 & 0.6052 \\
\hline & FST_2_3 & 0.1761 & $\left.0.00011^{* * *}\right)$ & $0.9580\left(^{*}\right)$ & 0.6289 \\
\hline Test quantities & VAR_S & 21.7561 & 0.7476 & 0.2538 & 0.6209 \\
\hline corresponding & VAR_1 & 9.3385 & 0.4861 & 0.3561 & 0.3598 \\
\hline to summary & VAR_2 & 9.5277 & 0.5232 & 0.1792 & 0.3748 \\
\hline statistics NOT & LIK_1_S & 38.5648 & 0.7867 & 0.4503 & 0.7240 \\
\hline used to & LIK_1_2 & 31.7504 & $\left.0.00011^{* * *}\right)$ & $1.0000\left(^{* * *}\right)$ & 0.7162 \\
\hline discriminate & LIK_2_1 & 32.1075 & $\left.0.00011^{* * *}\right)$ & $0.9850\left(^{*}\right)$ & 0.7836 \\
\hline among & H2P_S_1 & 0.7734 & 0.6563 & 0.8411 & 0.6115 \\
\hline scenarios and & H2P_S_2 & 0.7993 & 0.9231 & 0.8239 & 0.8664 \\
\hline compute & H2P_1_2 & 0.6020 & $0.0315\left(^{*}\right)$ & $0.9975(* *)$ & 0.7193 \\
\hline parameter & DAS_S_1 & 0.1329 & 0.2298 & 0.4582 & 0.2639 \\
\hline posterior & DAS_S_2 & 0.1099 & 0.0559 & 0.1681 & 0.0816 \\
\hline distributions & DAS_1_2 & 0.3402 & $1.0000\left(^{* * *}\right)$ & $0.0001\left(^{* * *}\right)$ & 0.2529 \\
\hline
\end{tabular}

Evolutionary scenarios 1, 2 and 3 are detailed in Figure 3. The single "pseudo-observed" test data set analyzed here was simulated under scenario 3. The probability ( $t_{\text {simulated }}<t_{\text {observed }}$ ) given for each test quantities $(t)$ was computed from 10,000 data sets simulated from the posterior distributions of parameters obtained under a given scenario. Corresponding tail-area probabilities, or $p$-values, of the test quantities $(t)$ can be easily obtained as Prob $\left(t_{\text {simulated }}<t_{\text {observed }}\right)$ and 1.0 - Prob $\left(t_{\text {simulated }}<t_{\text {observed }}\right)$ for Prob $\left(t_{\text {simulated }}<t_{\text {observed }}\right) \leq 0.5$ and $>0.5$, respectively [22]. The test quantities correspond to the summary statistics used to discriminate among scenarios and compute the posterior distributions of parameters or to other statistics. NAL_ $i=$ mean number of alleles in population $i$, HET_ $i$ $=$ mean expected heterozygosity in population $i$ [38], MGW_ $i=$ mean ratio of the number of alleles over the range of allele sizes [54], FST_i $j=F_{\mathrm{ST}}$ value between populations $i$ and $j$ [39], VAR $i=$ mean allelic size variance in population $i$, LIK $i \_j=$ mean individual assignment likelihoods of population $i$ assigned to population $j$ [22], H2P_i $j=$ mean expected heterozygosity pooling samples from populations $i$ and $j$, DAS $i j j=$ shared allele distance between populations $i$ and $j$ [55]. Populations $i$ and $j$ correspond to populations $S, 1$ or 2 in Figure $3 .{ }^{*}, *^{* *}{ }^{* * *}=$ tail-area probability $<0.05,<0.01$ and $<0.001$, respectively. Significant tailarea probabilities after applying the false discovery rate correction method of Benjamini and Hochberg [43] are given in bold italic characters.

We found that, depending on the parameter considered, cumulating the information provided by different markers translated into a decrease, an increase or, most frequently, an absence of noticeable variation of the RMAE values compared to that obtained with the most informative genetic marker (Figure 4 and see Additional file 2 for an illustration of the variation of RMAE values expected by chance between different replicates of 500 pseudo-observed data sets). Although each category of markers is different and genealogically independent, the genetic variation at these markers is constrained by the fact that they share the same evolutionary history (i.e. same historical and demographic parameters) so that information provided by each category is not expected to sum up. For all demographic parameters, the lowest RMAE values were obtained, however, when combining the three categories of markers; but in many cases, one or the other category (depending on the considered parameter), taken alone, provided almost the same precision.

We found that adding sequence data substantially improved the quality of the estimations of some parameters in comparison to results obtained with microsatellites only. This was particularly true for the most ancient divergence time $t 5$ for which RMAE values decreased by $41 \%, 36 \%$ and $47 \%$ when adding a single mtDNA sequence, five nuclear sequence and both types of sequences, respectively. Only small decreases of RMAE values were observed for moderately ancient events such as the divergence time $t 3$ and the admixture rate $r 2$. This result underlines the interest of using low mutating and seldom homoplasious sequence data for making inferences on ancient historical events. In agreement with this, the two RMAE values for $t 5$ obtained with the mtDNA data sets and the nuclear sequence data sets were lower than that obtained for 


\section{Type I error}

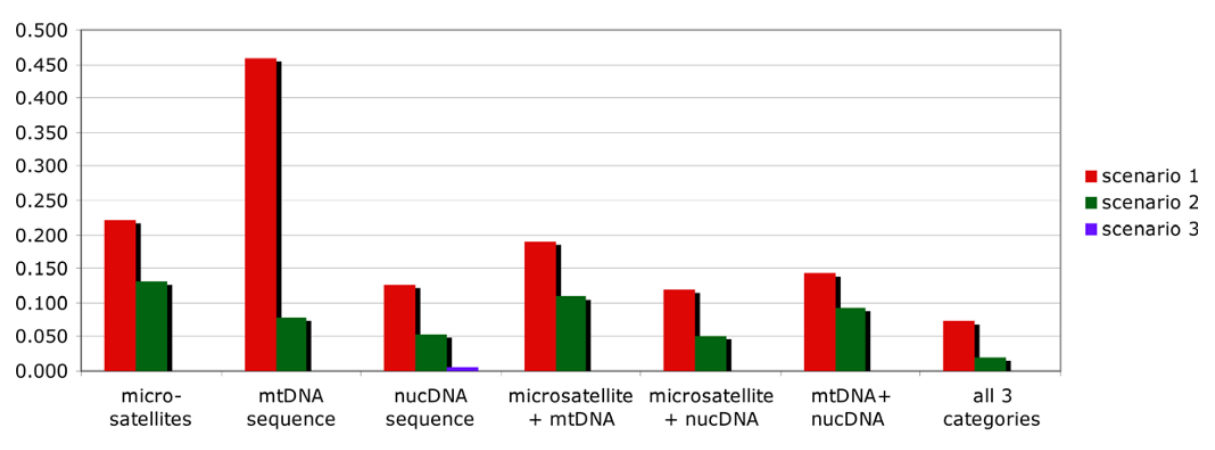

Type II error

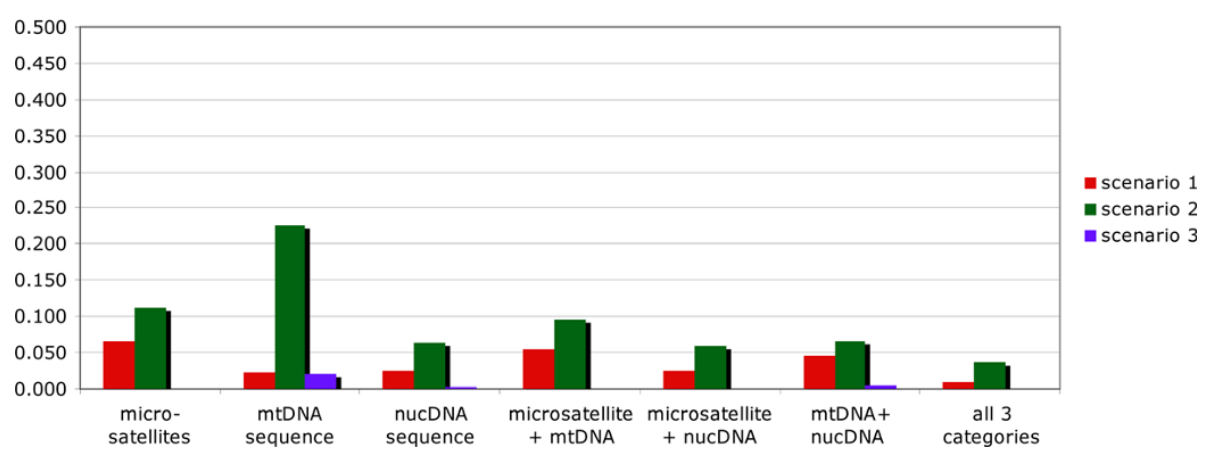

Figure 3 Confidence in discriminating evolutionary scenarios using microsatellite, mtDNA and/or nuclear DNA sequence data. The three compared scenarios are detailed in Figure 1. Type I error: exclude scenario $x$ when it is actually scenario $x$. Type II error: choose scenario $x$ when it is not scenario $x$. Results are based on 500 simulated data sets per scenario with parameter values drawn from the same distributions as the prior distributions given in the legend of Figure 1.

microsatellite loci only. Due to their mutation modalities (high mutation rates with allele size homoplasy and constraints [37]), it is not surprising that microsatellite loci performed poorly for ancient evolutionary events $[51,52]$. On the opposite, microsatellite markers provided substantially better estimation than mtDNA or nuclear sequence for the most recent admixture rate $(r 1)$. The RMAE values for the mtDNA sequence and the nuclear sequences were two to three times larger than those obtained with microsatellite only for this parameter. As a result, the addition of mtDNA or nuclear sequences to microsatellite data did not bring any progress in terms of RMAE for $r 1$. This result holds to a lesser extent for the effective population size $N$.

\section{Model checking}

When considering altogether the three scenarios in our model discrimination analysis, we found that our (single) pseudo-observed test data set generated under the unsampled population scenario (scenario 3 in Figure 2) was unambiguously assigned to the correct scenario with a high posterior probability $(\mathrm{p}=0.9967,95 \%$ CI $[0.9958,0.9976])$. When only scenarios 1 and 2 were proposed for posterior probability estimation then the same test data set generated under scenario 3 was assigned to the incorrect scenario 2 with a high posterior probability ( $\mathrm{p}=0.9999,95 \%$ CI $[0.9998,1.0000])$. Additional $\mathrm{ABC}$ treatments achieved on larger sets of pseudo-observed test data sets $(n=1,000)$ confirmed that if only the traditional independent and serial introduction scenarios are considered, a data set obtained under the unsampled population scenario will erroneously be chosen, with often a high posterior probability to one of the two competing scenario; scenario 2 is chosen for $55 \%$ and $63 \%$ of the data sets generated under scenario 3 when simulating test data sets using the same fixed parameter values than the above single test data set and when drawing parameter values in the same distribution than those chosen as priors, respectively.

Focusing on our single pseudo-observed test data set simulated under scenario 3 , we evaluated in details the 


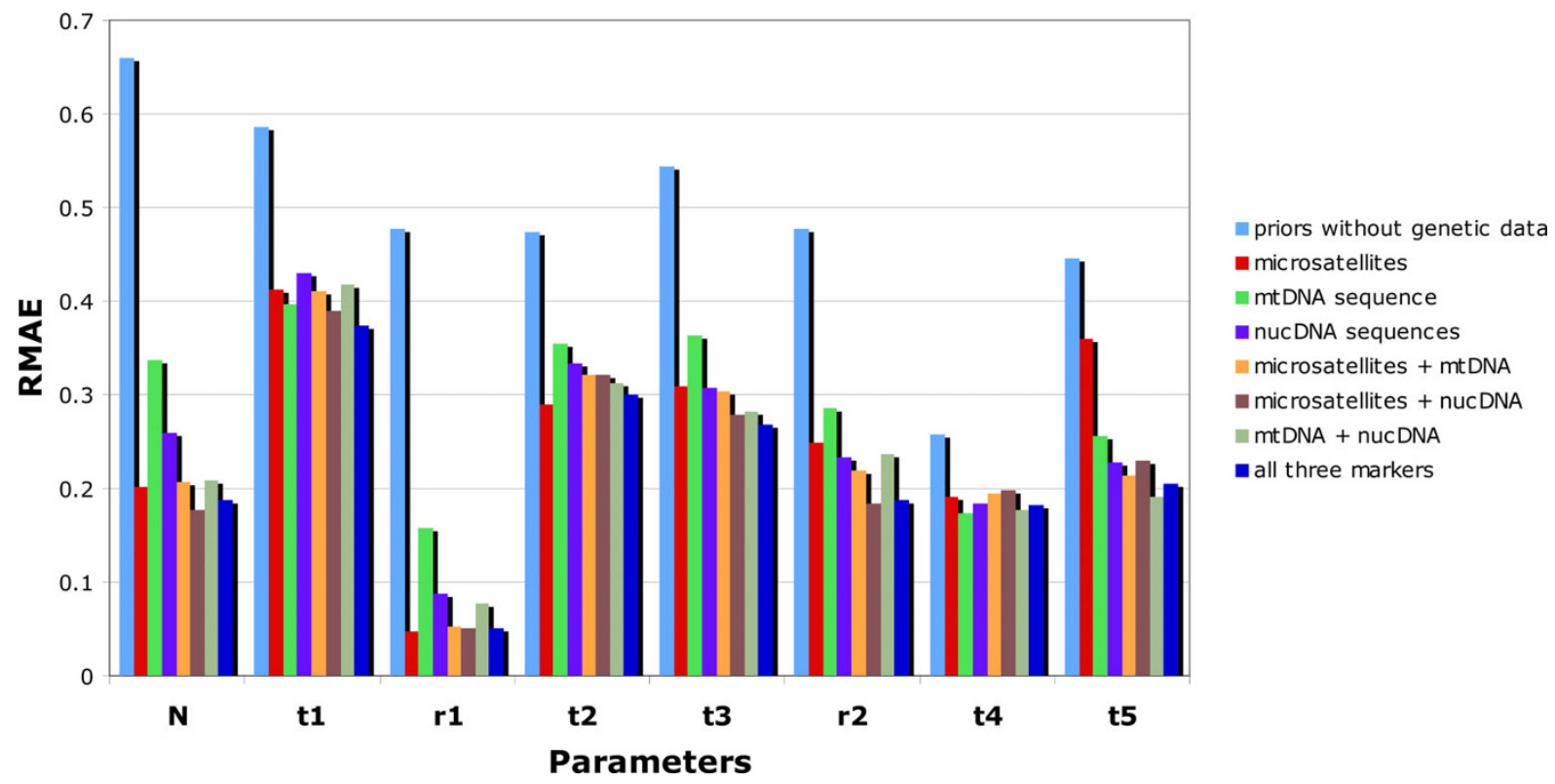

Figure 4 Precision in parameter estimation using microsatellite, mtDNA and/or nuclear DNA sequence data under scenario 1. Results are based on 500 pseudo-observed test data sets simulated and estimated under scenario 1 presented in Figure 1, with parameter values drawn from the same distributions as the prior distributions given in the legend of Figure 1. The demographic parameters $N, t 1, t 2, t 3, t 4, t 5, r 1$ and $r 2$ are detailed in Figure 1. RMAE: relative median absolute errors. The blue columns correspond to the "base-level" RMAE values obtained using only the prior information on parameters (no genetic data).

interest of the model checking option of DIYABC V1.0 to assess model misfit. We found that none of the twelve test quantities had low tail probability values when applying the model checking option to the (true) scenario 3 (last column of Table 1). In contrast, one to several test quantities had low tail-area probabilities (sometimes lower than $\mathrm{p}=0.001$ ) when applying the model checking option to (incorrect) scenarios 1 or 2, hence casting serious doubts on the adequacy of the tested model-posterior combination. We found some indication of a risk of over-estimating the quality of the fit by using as test quantities the same summary statistics already used during the inference steps (model discrimination and posterior estimation of parameters); see Table 1. The proportion of test quantities with low tailarea probabilities was indeed larger when using summary statistics not previously used for inference. A close examination of which summary statistics displayed low tail-area probabilities provides some insights on which aspects of the models 1 and 2 are problematic. In the studied case, outlying statistics correspond to an overestimated genetic differentiation in simulated data sets compared to the observed one between the introduced populations 1 and 2 for scenario 1, whereas it correspond to an underestimated genetic differentiation for scenario 2 . This pattern is in agreement with the specificities of the "true" scenario 3 (partial genealogical dependency between populations 2 and 3 through the unsampled population) relatively to scenario 1 (weak genealogical dependency between the independently introduced populations 2 and 3) and scenario 2 (strong genealogical dependency between the serially introduced populations 2 and 3); see Figure 3.

We further inspected the fit/misfit of models by performing several principal component analysis on the test quantities obtained with the different model -posterior combinations together with the pseudo-observed test data set simulated under the unsampled population scenario (Additional file 3). In agreement with the quantitative results summarized in Table 1, the PCA points of the test quantities obtained from the model-posterior combination corresponding to the (true) scenario 3 were nicely grouped and centred on the target point corresponding to the pseudo-observed test data set. This configuration holds when considering either previously used or unused $A B C$ summary statistics as test quantities. When considering scenarios 1 and 2, we found that the target point of the "pseudo-observed" test data set was positioned at best on the border of the cloud of PCA points of the test quantities corresponding to the summary statistics previously used for ABC analyses. Interestingly enough, the target point was clearly outside the cluster when considering unused summary statistics as test quantities.

The model checking analysis of other pseudo-observed test data sets provided results (quantitatively) similar to 
those presented in the Table 1 and Additional file 3 (results not shown).

\section{Conclusions}

The software DIYABC V1.0 offers a user-friendly interface allowing non-expert users to perform additional and more accurate inferences using $\mathrm{ABC}$ than its previous version. The new implementations allow the treatment of haploid in addition to diploid data and allow making inferences from DNA sequence data (without recombination) in addition or separately to microsatellite data. The possibility of mixing different types of molecular markers (including autosomal, X or Y-linked loci, and mtDNA loci) should prove useful when considering complex evolutionary scenarios involving both recent and ancient historical events. Finally, DIYABC V1.0 offers non-specialist users a handy way to achieve model checking computation (i.e. the assessment of the "goodness-of-fit" of a model - posterior combination with respect to a target data set), a feature of $A B C$ analysis that has been so far neglected. These new software developments significantly enlarge the tool box available to biologists to make $\mathrm{ABC}$ inferences on more complex and hence more realistic demographic processes that have acted on natural populations. The main limitations of the current version of DIYABC are the assumed absence of migration among populations after they have diverged, the impossibility to consider other reproduction systems than standard sexuality as well as evolutionary neutrality of markers. Next developments will aim at progressively removing these limitations.

\section{Additional material}

Additional file 1: Pre-evaluation of model-prior combinations: two examples. Pre-evaluation of model-prior combinations: example 1 A single test pseudo-observed data set (10 microsatellite loci) was first simulated under a model of a single population (sample size of 30 diploid individuals) with effective size $N=10,000$. Microsatellite loci were assumed to follow a generalized stepwise mutation model (GSM [37]) with a mean mutation rate (mean $\mu$ ) equal to $5 \times 10^{-4}$ and a mean parameter of the geometric distribution of the length in number of repeats of mutation events (mean $P$ ) equal to 0.22 . Each locus was given a possible range of 40 contiguous allelic states and was characterized by individual $\mu_{l o c}$ and $P_{l o c}$ values drawn from Gamma(mean $=$ mean $\mu$ and shape $=2)$ and $G a m m a(m e a n=$ mean $P$ and shape $=2)$ distributions, respectively [12]. For $A B C$ analysis of the test data set, we used the same population and marker models, and prior distributions of demographic parameters were as followed: Uniform[10; 1000] (figure A) or Uniform [2000; 20000] (figure B) for $N$, Uniform $\left[10^{-4} ; 10^{-3}\right.$ ] and Uniform $[0.1 ; 0.3]$ for mean $\mu$ and mean $P$, respectively. We choose three summary statistics (s): mean number of alleles, mean expected heterozygosity [38] and mean allele size variance per population. PCA on summary statistics (A and B) and probability ( $s_{\text {simulated }}<S_{\text {observed }}$ ) for each summary statistics (C) were computed from 10,000 simulations, randomly drawing parameter values from priors. Pre-evaluation of model-prior combinations: example 2. A single pseudo-observed test data set (10 microsatellite loci) was first simulated under a model of two populations (sample size of 30 diploid individuals per population) splitting at time $t=10,000$ generations from an ancestral population, without subsequent migration. For all populations the effective size was $N=1,000$. For $A B C$ analysis of the test data set, we used the same population and marker models, and prior distributions of demographic parameters were as followed: Uniform[100; 1000] (figure D) or Uniform[2000; 20000] (figure E) for t, and Uniform[100; 2000] for $N$. The mutation model and priors for microsatellite markers are the same as in example 1. We choose eight summary statistics (s): mean number of alleles, mean expected heterozygosity [38] and mean allele size variance of each population sample, and $F_{\text {ST }}$ values and genetic distances $(\delta \mu)^{2}$ between pairs of populations [39,40]. PCA on summary statistics ( $D$ and $E$ ) and probability $\left(s_{\text {simulated }}<s_{\text {observed }}\right.$ ) for each of the summary statistics (F) were computed from 10,000 simulaxtions, randomly drawing parameter values from priors.

\section{Additional file 2: Evaluation of the variation of RMAE values} expected by chance between different replicates of 500 pseudoobserved data sets. relative median absolute errors (RMAE) were computed for 10 replicates of 500 pseudo-observed data sets simulated under scenario 1. The data sets include 20 (independent) microsatellite loci and were generated under scenario 1 presented in Figure 1. Parameter values were drawn from the same distributions than the prior distributions given in the legend of Figure 1. The demographic parameters $N, t 1, t 2, t 3, t 4, t 5, r 1$ and $r 2$ are detailed in Figure 1 . Standard deviation of RMAE values were equal to $0.009,0.019,0.004,0.017,0.012$, 0.013 and 0.014 for $N, t 1, t 2, t 3, t 4, t 5, r 1$ and $r 2$, respectively. Similar levels of RMAE variation among replicates of 500 pseudo-observed data sets were obtained for other categories of genetic markers ( $m$ tDNA and nuclear sequences) and combinations of categories of markers (results not shown).

Additional file 3: Principal component analysis of test quantities when processing model checking for the introduction scenarios 1 , 2 and 3. The scenarios 1, 2 and 3 are detailed in Figure 2. The pseudoobserved test data set analyzed here was simulated under scenario 3. PCA were processed on the test quantities corresponding to the summary statistics used to discriminate among scenarios and compute the posterior distributions of parameters (a) or on other statistics (b). The summary statistics used as test quantities are detailed in the legend of Table 1.

\section{Acknowledgements}

We thank Gaël Kergoat, Renaud Vitalis and Alexandre Dehne-Garcia for useful discussions. This research was financially supported by the French Agence Nationale de la Recherche grants ANR-09-BLAN-0145-01 to AE, JMC and VR and ANR-07-BDIV-003 (Emerfundis project) to VR.

\section{Author details}

${ }^{1}$ INRA, UMR CBGP (INRA/IRD/Cirad/Montpellier SupAgro), Campus international de Baillarguet, CS 30016, F-34988 Montferrier-sur-Lez cedex, France. ${ }^{2} \mathrm{CIRAD}$, Unité Mixte de Recherche-Biologie et Génétique des Interaction Plante-Parasite, F-34398 Montpellier, France.

\section{Authors' contributions}

$J M C$ and $A E$ carried out the analyses, wrote the paper and jointly developed the software DIYABC V1.0 with VR. All authors read and approved the final manuscript.

Received: 2 April 2010 Accepted: 28 July 2010 Published: 28 July 2010

\section{References}

1. Beaumont MA, Rannala B: The Bayesian revolution in genetics. Nat Rev Genet 2004, 5:251-261.

2. Excoffier L, Heckel G: Computer programs for population genetics data analysis: a survival guide. Nat Rev Genet 2006, 7:745-758.

3. Beaumont MA, Zhang WY, Balding DJ: Approximate Bayesian computation in population genetics. Genetics 2002, 162:2025-2035

4. Bertorelle $G$, Bonazzo $A$, Mona $S: A B C$ as a flexible framework to estimate demography over space and time: some cons, many pros. Mol Ecol 2010.

5. Csilléry K, Blum MGB, Gaggiotti O, François O: Approximate Bayesian Computation (ABC) in practice. Trends Ecol Evol 2010, 25:410-418. 
6. Estoup A, Beaumont M, Sennedot F, Moritz C, Cornuet JM: Genetic analysis of complex demographic scenarios: Spatially expanding populations of the cane toad, Bufo marinus. Evolution 2004, 58:2021-2036.

7. Fagundes NJR, Ray N, Beaumont MA, Neuenschwander S, Salzano FM, Bonatto SL, Excoffier L: Statistical evaluation of alternative models of human evolution. Proc Nat Acad Sci USA 2007, 104:17614-17619.

8. Jakobsson M, Hagenblad J, Tavaré S, Säll T, Halldén C, Lind-Halldén C, M N: A recent unique origin of the allotetraploid species Arabidopsis suecica: evidence from nuclear DNA markers. Mol Biol Evol 2006, 23:1217-1231.

9. Neuenschwander S, Largiader CR, Ray N, Currat M, Vonlanthen P, Excoffier $\mathrm{L}$ : Colonization history of the Swiss Rhine basin by the bullhead (Cottus gobio): inference under a Bayesian spatially explicit framework. Mol Ecol 2008, 17:757-772.

10. Patin E, Laval G, Barreiro LB, Salas A, Semino O, Santachiara-Benerecetti S, et al: Inferring the demographic history of African farmers and Pygmy hunter-gathers using a multilocus resequencing data set. PLoS Genet 2009, 5:e1000448.

11. Rosenblum EB, Hickerson MJ, Moritz C: A multilocus perspective on colonization accompanied by selection and gene flow. Evolution 2007, 61:2971-2985.

12. Verdu P, et al: Origins and Genetic Diversity of Pygmy Hunter-Gatherers from Western Central Africa. Current Biology 2009, 19:1-7.

13. Lombaert E, Guillemaud T, Cornuet J-M, Malausa T, Facon B, Estoup A: Bridgehead effect in the worldwide invasion of the biocontrol harlequin ladybird. PLOS ONE 2010, 5:e9743.

14. Luciania F, Sisson SA, Jiang H, Francis AR, Tanaka MM: The epidemiological fitness cost of drug resistance in Mycobacterium tuberculosis. Proc Natl Acad Sci USA 2009, 106:14711-14715.

15. Ratmann O, Andrieu C, Wiuf C, Richardson S: Model criticism based on likelihood-free inference, with an application to protein network evolution. Proc Natl Acad Sci USA 2009, 106:10576-10581.

16. Beaumont MA, Cornuet JM, Marin JM, Robert C: Adaptive approximated Bayesian computation. Biometrika 2009, 96:983-990.

17. Bortot $\mathrm{P}$, Coles S, Sisson S: Inference for stereological extremes. J Am Statist Assoc 2007, 102:84-92.

18. Leuenberger $C$, Wegmann D: Bayesian Computation and model selection without likelihoods. Genetics 2009, 184:243-252.

19. Marjoram P, Molitor J, Plagnol V, Tavaré S: Markov chain Monte Carlo without likelihoods. Proc Natl Acad Sci USA 2003, 100:15324-15328

20. Lopes JS, Beaumont MA: ABC: a useful Bayesian tool for the analysis of population data. Inf Genet Evol 2009, 10:825-832.

21. Beaumont MA, Nielsen R, Robert $C$, Hey J, Gaggiotti O, Knowles L, et al: In defence of model-based inference in phylogeography. Mol Ecol 2010, 9:436-446

22. Gelman A, Carlin JB, Stern HS, et al: Bayesian Data Analysis Chapman and Hall, New York 1995

23. Ingvarsson PK: Multilocus patterns of nucleotide polymorphism and the demographic history of Populus tremula. Genetics 2008, 180:329-340.

24. Pascual M, Chapuis MP, Mestres F, Balanya J, Huey RB, Gilchrist GW, et al: Introduction history of Drosophila subobscura in the New World: a microsatellite-based survey using ABC methods. Mol Ecol 2007, 16:3069-3083.

25. Ghirotto S, Mona S, Benazzo A, Paparazzo F, Caramelli D, Barbujani G: Inferring genealogical processes from patterns of bronze-age and modern DNA variation in Sardinia. Mol Biol Evol 2010, 27:875-886.

26. Cornuet JM, Santos F, Beaumont MA, Robert CP, Marin J-M, Balding DJ, Guillemaud T, Estoup A: Inferring population history with DIYABC: a userfriendly approach to Approximate Bayesian Computation. Bioinformatics 2008, 24:2713-2719.

27. Foll M, Gaggiotti O: A genome-scan method to identify selected loci appropriate for both dominant and codominant markers: a Bayesian perspective. Genetics 2008, 180:977-993.

28. Hickerson MJ, Meyer CP: Testing comparative phylogeographic models of marine vicariance and dispersal using a hierarchical Bayesian approach. BMC Evol Biol 2008, 8:322.

29. Jobin MJ, Moutain JL: REJECTOR: software for population history inference from genetic data via a rejection algorithm. Bioinformatics 2008, 24:2936-2937.

30. Tallmon DA, Koyuk A, Luikart G, Beaumont MA: ONESAMP: a program to estimate effective population size using approximate Bayesian computation. Mol Ecol Res 2008, 8:299-301.
31. Lopes JS, Balding D, Beaumont MA: PopABC: a program to infer historical demographic parameters. Bioinformatics 2009, 25:2747-2749.

32. Wegmann D, Leuenberger C, Neuenschwander S, Excoffier L: ABCtoolbox: aversatile toolkit for approximate Bayesian computations. $B M C$ Bioinformatics 2010, 11:116-122

33. Jukes TH, Cantor CR: Evolution of protein molecules. Mammalian protein metabolism Academic Press, New YorkMunro HN 1969, 21-123.

34. Kimura M: A simple method for estimating evolutionary rate of base substitution through comparative studies of nucleotide sequences. J Mol Evol 1980, 16:111-120.

35. Hasegawa M, Kishino $H$, Yano T: Dating the human-ape splitting by a molecular clock of mitochondrial DNA. J Mol Evol 1985, 22:160-174.

36. Tamura $K$, Nei M: Estimation of the number of nucleotide substitutions in the control region of mitochondrial DNA in humans and chimpanzees. Mol Biol Evol 1993, 10:512-526.

37. Estoup A, Jarne P, Cornuet JM: Homoplasy and mutation model at microsatellite loci and their consequences for population genetics analysis. Mol Ecol 2002, 11:1591-1604.

38. Nei M: Molecular Evolutionary Genetics Columbia University Press, New York 1987

39. Weir BS, Cockerham C: Estimating F-statistics for the analysis of population structure. Evolution 1984, 38:1358-1370.

40. Goldstein DB, Linares AR, Cavalli-Sforza LL, Feldman MW: An evaluation of genetic distances for use with microsatellite loci. Genetics 1995, 139:463-471.

41. Choisy M, Franck P, Cornuet JM: Estimating admixture proportions with microsatellites: comparison of methods based on simulated data. $\mathrm{Mol}$ Ecol 2004, 13:955-968.

42. Hudson RR, Slatkin M, Maddison WP: Estimation of levels of gene flow from DNA sequence data. Genetics 1992, 132:583-589.

43. Benjamini $Y$, Hochberg $Y$ : Controlling the false discovery rate:/a practical and powerful approach to multiple testing. J R Stat Soc B 1995, 57:289-300.

44. Verhoeven KJF, Simonsen KL, McIntyre LM: Implementing false discovery rate control: increasing your power. Oikos 2005, 108:643-647.

45. Dey DK, Rao CR: Bayesian thinking: modelling and computation Handbook of Statistics 25. Elsevier London 2005.

46. Miller N, Estoup A, Toepfer S, Bourguet D, Lapchin L, Derridj S, et al: Multiple transatlantic introductions of the western corn rootworm. Science 2005, 310:992.

47. Guillemaud T, Beaumont MA, Ciosi M, Cornuet JM, Estoup A: Inferring introduction routes of invasive species using approximate Bayesian computation on microsatellite data. Heredity 2010, 104:88-99.

48. Roman J: Diluting the founder effect: cryptic invasions expand a marine invader's range. Proc $R$ Soc B 2006, 273:2453-2459.

49. Saltonstall $K$ : Cryptic invasion by a non-native genotype of the common reed, Phragmites australis, into North America. Proc Natl Acad Sci USA 2002, 99:2445-2449.

50. Degnan JH, Rosenberg NA: Gene tree discordance, phylogenetic inference and the multispecies coalescent. Tr Ecol Evol 2009, 24:332-340.

51. Takezaki N, Nei M: Genetic distances and reconstruction of phylogenetic trees from microsatellite DNA. Genetics 1996, 144:389-399.

52. Pollock DD, Bergman A, Feldman MW, Goldstein DB: Microsatellite behavior with range constraints: parameter estimation and improved distances for use in phylogenetic reconstruction. Theoret Pop Biol 1998, 53:256-271.

53. Haag-Liautard C, Coffey N, Houle D, Lynch M, Charlesworth B, Keightley PD: Direct estimation of the mitochondrial DNA mutation rate in Drosophila melanogaster. PLoS Biol 2008, 6:e204.

54. Excoffier L, Estoup A, Cornuet JM: Bayesian analysis of an admixture model with mutations and arbitrarily linked markers. Genetics 2005, 169:1727-1738.

55. Jin L, Chakraborty R: Estimation of Genetic Distance and Coefficient of Gene diversity from Single-Probe Multilocus DNA Fingerprinting Data. Mol Biol Evol 1993, 11:120-127.

doi:10.1186/1471-2105-11-401

Cite this article as: Cornuet et al: Inference on population history and model checking using DNA sequence and microsatellite data with the software DIYABC (v1.0). BMC Bioinformatics 2010 11:401. 